\title{
Detección de trastornos temporomandibulares en estudiantes de la Universidad Privada de Huancayo Franklin Roosevelt - 2018
}

\author{
Detection of temporomandibular disorders in students of Franklin Roosevelt Private University of \\ Huancayo - 2018 \\ Luis Alberto Cueva Buendía ${ }^{1, a}$, Jhon Rondinel Berna 1,a, Juan Jesús Buendía Suazo ${ }^{1, a}$, \\ Mayk Erick Peralta Peña ${ }^{1}$, Marisol Yeni Quispe Mayta ${ }^{1}$, Lizbeth Jhancarla Aguirre Quispe ${ }^{1}$
}

\section{RESUMEN}

Objetivos: Describir los trastornos temporomandibulares, más prevalentes, según el índice de Helkimo en los estudiantes de la Universidad Privada de Huancayo Franklin Roosevelt, evaluados a inicio del semestre académico 2018-I. Material y Métodos: Se usó como método general, el científico y como específico, el observacional. Tipo de investigación básica, diseño descriptivo simple. La población fue de 2276 estudiantes del I al X ciclo de ambos sexos y matriculados en sus respectivos ciclos, pertenecientes de las escuelas profesionales de la Universidad Privada de Huancayo Franklin Roosevelt. La muestra fue 329 estudiantes seleccionados con muestreo probabilístico estratificado. La recolección de datos se realizó a través de la técnica de observación y el instrumento la ficha clínica a través del test de Helkimmo. Los métodos que se utilizaron para el análisis y procesamiento de los datos fue a través del software SPSS V 20 y Excel 2010. Resultados: Los resultados se presentaron en cuadros, tablas y gráficos de frecuencias y porcentajes. Se evidenció que el "trastorno moderado" alcanzó el 55,9\% de la muestra, el "trastorno severo grado I" alcanzó el 38,9\%, con "función normal" el 2,4\%, "trastorno severo grado III" el 1,2\%, "trastorno severo grado II el 0,9\% y trastorno leve el 0,6\%. Conclusiones: Se ha encontrado en la detección de los trastornos temporomandibulares, que el "trastorno moderado" es el más prevalente con el 55,9\% de la muestra, seguido del "trastorno severo grado I" con un 38,9\%, en los estudiantes de la universidad privada de Huancayo Franklin Roosevelt, evaluados en el semestre académico 2018-I, con el test de Helkimo.

PALABRAS CLAVES: articulación temporomandibular, trastorno temporomandibular.

\section{SUMMARY}

Objectives: To describe the temporomandibular disorders, most prevalent, according to the Helkimo index in the students of Franklin Roosevelt, private university of Huancayo, evaluated at the beginning of the academic semester 2018-I. Material and Methods: we used the scientific method in general and the observational in specific cases. Type of basic research simple descriptive design was used. The population was 2276 students from I to X cycle of both sexes and enrolled in their respective cycles, belonging to the professional schools of the private university of Huancayo Franklin Roosevelt. The sample was 329 selected students with stratified probabilistic sampling. Data collection was carried out through the observation technique and the instrument the clinical record through the Helkimmo test. The methods used for data analysis and processing were through the SPSS V 20 software and 
Excel 2010. Results: The results were presented in tables, charts and graphs of frequencies and percentages. It was evidenced that "moderate disorder" reached $55.9 \%$ of the sample, the "severe disorder grade I" reached $38.9 \%$, with "normal function" the $2.4 \%$, "severe disorder grade III" the $1.2 \%$, "grade II severe disorder $0.9 \%$ and mild disorder $0.6 \%$. Conclusions: It has been found in the detection of temporomandibular disorders, that "moderate disorder" is the most prevalent with 55.9 percent of the sample, followed by "severe disorder grade I" with 38.9 percent, in students of Franklin Roosevelt private University of Huancayo, evaluated in the academic semester 2018-I, with the Helkimo test.

KEYWORDS: temporomandibular articulation, temporomandibular disorder.

\section{INTRODUCCIÓN}

Los trastornos temporomandibularares (TTM), abarcan consigo diversas condiciones disfuncionales dolorosas que incluyen signos y síntomas. Estos desórdenes provienen de varias estructuras del sistema estomatognático como pueden ser las articulaciones temporomandibulares (ATM) con sus componentes capsulares y ligamentos, los músculos y en etapas más avanzadas pueden estar comprometidos los componentes óseos. Las personas con TTM presentan con mayor frecuencia episodios de dolor muscular y anomalías en el movimiento mandibular (1).

La gran mayoría de autores coinciden que en un inicio los pacientes refieren que no han buscado atención médica porque sus signos y síntomas se hallan dentro de parámetros que les permiten llevar una calidad de vida considerada normal y aceptable.

Puesto que dependiendo de la etapa en que se encuentre el TTM, muchas veces es asintomático y los pacientes que buscan asistencia lo hacen generalmente cuando se presenta algún dolor orofacial, que puede confundirse con afecciones del oído, de la cabeza, etc. Pero debemos tener en cuenta que los TTM representan el origen principal del dolor orofacial después del dolor dental (2).

Por esta razón el presente trabajo de investigación tuvo como problema general: ¿Cuáles son los trastornos temporomandibulares más prevalentes, según el índice de Helkimo en los estudiantes de la Universidad Privada de Huancayo Franklin Roosevelt, evaluados a inicio del semestre académico 2018I?, y el objetivo general planteado fue: describir los trastornos témporomandibulares, más prevalentes, según el índice de Helkimo en los estudiantes de la Universidad Privada de Huancayo Franklin Roosevelt, evaluados a inicio del semestre académico 2018-I.
Por consecuencia la justificación principal de este estudio fue teórica porque no existen trabajos de investigación en la institución universitaria donde se hizo el presente estudio.

La presente investigación tuvo el propósito de evaluar los problemas asociados con la articulación témporomandibular (ATM) y la musculatura de la mandíbula, así como todas las estructuras que tienen una relación directa o indirecta con la misma como los dientes, la cabeza y cervicales en los estudiantes de todas las carreras profesionales de la Universidad Privada Franklin Roosevelt, al inicio del semestre académico 2018-I; con la intención de identificar, prevenir y anticiparnos a la instalación y progresión de dichos trastornos que traen consigo cefaleas, cefalgia, dolor, ruido en las articulaciones, trabas articulares en los movimientos de apertura y cierre de la boca, dolor miofascial, contractura de músculos de cabeza y cuello, etc., que puede indudablemente influenciar en el abandono o deserción estudiantil, complicaciones en la necesidad de tratamientos médicos, y confusión en el tratamiento específico.

Para que, reconociendo, diagnosticando y planificando tempranamente estos signos y síntomas se brinde un tratamiento correcto, que asegure el bienestar y confort en ellos.

\section{MATERIAL Y MÉTODOS}

El método general utilizado fue el científico y el específico el observacional, corresponde al tipo de investigación básica, diseño descriptivo simple. La población fue de 2276 estudiantes del I al X ciclo de ambos sexos y matriculados en sus respectivos ciclos, pertenecientes de las escuelas profesionales de la Universidad Privada de Huancayo Franklin Roosevelt.

La muestra fue de 329 estudiantes seleccionados con muestreo probabilístico estratificado. La 
recolección de datos se realizó a través de la técnica de observación y el instrumento la ficha clínica a través del test de Helkimo. Los métodos que se utilizaron para el análisis y procesamiento de los datos fue a través del software SPSS V 20 y Excel 2010, los resultados se presentaron en cuadros, tablas y gráficos de frecuencias y porcentajes.

\section{RESULTADOS}

Se encontró que el 1 "trastorno moderado" es el más prevalente con el $55,9 \%$, el "trastorno severo grado I" alcanzó el 38,9\%, con "función normal" el $2,4 \%$, "trastorno severo grado III" el 1,2\%, "trastorno severo grado II el $0,9 \%$ y trastorno leve el $0,6 \%$ (tabla $1)$.

De acuerdo a las características de la muestra se encontró en correspondencia a la edad el "trastorno moderado", presente en un 40,12\% en el grupo de 15 a 34 años (tabla 2), según el sexo en el femenino en un $42,5 \%$, (tabla 3 ) y según la escuela profesional está presente en un $27,4 \%$ en Ciencias Farmacéuticas y Bioquímica (tabla 4).

Tabla 1. Tipo de trastorno temporomandibular

\begin{tabular}{llcccc}
\hline \multicolumn{5}{c}{ TIPO DE TRASTORNO TÉMPOROMANDIBULAR } \\
\hline \multirow{6}{*}{ Válidos } & Frecuencia & Porcentaje & Porcentaje válido & Porcentaje acumulado \\
\hline \multirow{4}{*}{ Paciente con función normal } & 8 & 2,4 & 2,4 & 2,4 \\
& Trastorno Leve & 2 &, 6 &, 6 & 3,0 \\
& Trastorno Moderado & 184 & 55,9 & 55,9 & 59,0 \\
& Trastorno Severo grado I & 128 & 38,9 & 38,9 & 97,9 \\
& Trastorno Severo grado II & 3 &, 9 &, 9 & 98,8 \\
& Trastorno Severo grado III & 4 & 1,2 & 1,2 & 100,0 \\
& Total & 329 & 100,0 & 100,0 & \\
\hline
\end{tabular}

Tabla 2. Tabla de contingencia tipo de trastorno temporomandibular * edad

\begin{tabular}{llcccc}
\hline & & \multicolumn{3}{c}{ EDAD } & \multirow{2}{*}{ Total } \\
\cline { 3 - 5 } & & $\mathbf{1 5 - 2 4}$ años & $\mathbf{2 5 - 3 4}$ años & $>\mathbf{3 4}$ años & \\
\hline \multirow{2}{*}{ TIPO DE TRASTORNO } & Paciente con función normal & 7 & 1 & 0 & 8 \\
TÉMPOROMANDIBULAR & Trastorno Leve & 2 & 0 & 0 & 2 \\
& Trastorno Moderado & 132 & 44 & 8 & 184 \\
& Trastorno Severo grado I & 97 & 27 & 4 & 128 \\
& Trastorno Severo grado II & 0 & 3 & 0 & 3 \\
Total & Trastorno Severo grado III & 4 & 0 & 0 & 4 \\
& & 242 & 75 & 12 & 329 \\
\hline
\end{tabular}

Tabla 3. Tabla de contingencia tipo de trastorno témporomandibular * sexo

\begin{tabular}{llccc}
\hline & & \multicolumn{2}{c}{ SEXO } & \multirow{2}{*}{ Total } \\
\cline { 3 - 4 } & & Masculino & Femenino & \\
\hline \multirow{3}{*}{ TIPO DE TRASTORNO } & Paciente con función normal & 0 & 8 & 8 \\
TÉMPOROMANDIBULAR & Trastorno Leve & 1 & 1 & 2 \\
& Trastorno Moderado & 44 & 140 & 184 \\
& Trastorno Severo grado I & 19 & 109 & 128 \\
& Trastorno Severo grado II & 0 & 3 & 3 \\
Total & Trastorno Severo grado III & 3 & 1 & 4 \\
\hline
\end{tabular}


Tabla 4. Tabla de contingencia tipo de trastorno témporomandibular * escuela profesional

\begin{tabular}{|c|c|c|c|c|c|c|c|}
\hline & \multicolumn{5}{|c|}{ ESCUELA PROFESIONAL } & \multirow[b]{2}{*}{ Total } \\
\hline & & Administración & $\begin{array}{c}\text { Ciencias } \\
\text { farmacéuticas } \\
\text { y bioquímica }\end{array}$ & Enfermería & Estomatología & Obstetricia & \\
\hline \multirow{6}{*}{$\begin{array}{l}\text { TIPO DE } \\
\text { TRASTORNO } \\
\text { TÉMPORO_- } \\
\text { MANDIBULAR }\end{array}$} & $\begin{array}{l}\text { Paciente } \\
\text { con función } \\
\text { normal }\end{array}$ & 0 & 4 & 2 & 0 & 2 & 8 \\
\hline & $\begin{array}{l}\text { Trastorno } \\
\text { Leve }\end{array}$ & 0 & 1 & 1 & 0 & 0 & 2 \\
\hline & $\begin{array}{l}\text { Trastorno } \\
\text { Moderado }\end{array}$ & 25 & 90 & 31 & 18 & 20 & 184 \\
\hline & $\begin{array}{c}\text { Trastorno } \\
\text { Severo grado I }\end{array}$ & 19 & 31 & 40 & 16 & 22 & 128 \\
\hline & $\begin{array}{c}\text { Trastorno } \\
\text { Severo grado } \\
\text { II }\end{array}$ & 0 & 1 & 0 & 1 & 1 & 3 \\
\hline & $\begin{array}{c}\text { Trastorno } \\
\text { Severo grado } \\
\text { III }\end{array}$ & 0 & 1 & 2 & 0 & 1 & 4 \\
\hline Total & & 44 & 128 & 76 & 35 & 46 & 329 \\
\hline
\end{tabular}

\section{DISCUSIÓN}

Después de haber realizado el análisis de los resultados de las pruebas estadísticas y la interpretación de los mismos, se presenta la siguiente discusión: La Asociación Dental Americana (ADA por sus siglas en inglés) ha adoptado el término de trastornos temporomandibulares para determinar a un grupo heterogéneo de condiciones clínicas caracterizadas por dolor y disfunción del sistema masticatorio (3). El término trastornos temporomandibulares engloba las disfunciones de la articulación temporomandibular, así como las alteraciones funcionales del sistema masticatorio.

Dawson define que los TTM son cualquier trastorno que afecta o está afectando por deformidad, enfermedad, desalineamiento o disfunción de la articulación temporomandibular. Esto incluye la desviación oclusal de la ATM y las respuestas asociadas a la musculatura (4). Los TTM comprenden una serie de alteraciones intraarticulares, periarticulares, sistémicas, aunque se puede manifestar como combinaciones entre ellas (5).

Primero es necesario indicar que la investigación se realizó en 329 estudiantes de las escuelas profesionales de la Universidad Privada de Huancayo Franklin Roosevelt, cuya distribución de edades fue: entre 15 a 24 años el 73,6\% de la muestra, entre 25 y 34 años el 22,8\% y mayores de 34 años fueron el 3,6\%. En cuanto al sexo el $76,6 \%$ fueron de sexo femenino y el $20,4 \%$ de sexo masculino, y en cuanto a las Escuelas Profesionales de Administración se consideró un $13,4 \%$, de Ciencias Farmacéuticas y Bioquímica el $38,9 \%$, Enfermería el $23,1 \%$, Estomatología el 10,6\% y Obstetricia el $14 \%$ de la muestra de estudio.

A continuación, se presentan la discusión, respecto al objetivo general que fue: describir los trastornos témporomandibulares, más prevalentes, se ha encontrado que el "trastorno moderado" es el más prevalente con el $55,9 \%$ de la muestra, seguido del "trastorno severo grado I" con un 38,9\% con 128 datos, en los estudiantes de la Universidad Privada de Huancayo Franklin Roosevelt, evaluados en el semestre académico 2018-I, con el Test de Helkimo.

Trabajos similares realizados por Silva, quien evaluó a 20 pacientes con TTM (19 mujeres y 1 varón) entre los 17 a 43 años del Programa de la Facultad de Odontología de la Universidad Federal de Uberlândia (FOUFU) - Brasil. Examinó a los pacientes utilizando el índice de Helkimo para hallar trastornos témporomandibulares (TTM). Concluyendo que un $35 \%$ de los pacientes presentaban dolor severo, $40 \%$ dolor moderado y el 25\% dolor leve (6). También está el trabajo realizado por Gonçalves, quien efectuó un estudio a 40 pacientes de 20 y 40 años del Departamento 
de Odontología de la Universidad Estatal Paraíba - Brasil. El examen fue extraoral e intraoral con el fin de hallar trastornos témporomandibulares en los pacientes. Concluyó que un $45 \%$ de pacientes presentaba dolor en las articulaciones y un $12,5 \%$ dolor muscular (7).

Otro trabajo que utilizó el mismo Índice de Helkimo, aunque en otro tipo de población fue el de Hormiga, quien evaluó a 189 estudiantes entre 16 años y 34 años ambos sexos de las áreas de salud: Odontología, Fisioterapia y Fonoaudiología del Área Metropolitana de Bucaramanga, para determinar trastornos témporomandibulares; se aplicó el Índice de Helkimo, obteniendo como resultado que la prevalencia de síntomas y signos de trastorno témporomandibular fue de $61,4 \%$ y $63,5 \%$ respectivamente (8). Del mismo modo tenemos el trabajo de Jiménez en el cual se examinaron a 1201 personas entre 15 años a más de ambos sexos de Ciudad de La Habana; el estudio consistió en anamnesis y examen clínico para hallar trastornos temporomandibulares, se usó el Índice de Helkimo, obteniendo como resultado que el $47,33 \%$ presentó signos clínicos de trastornos temporomandibulares siendo mayor la prevalencia de signos en el sexo femenino y proporcional con la edad, con mayor frecuencia de trastornos temporomandibular leve (9).

El trabajo de Padilla tuvo como objetivo determinar la prevalencia de signos, síntomas de los trastornos temporomandibulares (TTM) y grados según Índice de Helkimo, la investigación se realizó en 61 pacientes de 15 a 67 años de ambos sexos de la Clínica Odontológica de la Universidad Privada Norbert Wiener, Lima 2010, llegó a la conclusión de que la prevalencia de grados según el Índice de Helkimo y sexo, se presentó en 22 mujeres $(62,9 \%)$ y 17 hombres $(65,4 \%)$, diferencia que no fue estadísticamente significativa $(\mathrm{p}>0,05)$, la prevalencia de grados según Índice de Helkimo y edad se presentó en 39 pacientes $(63,9 \%)$ con trastorno témporomandibular leve (10).

Tenemos también a Limaylla, quien seleccionó de manera no probabilística y por conveniencia a 51 personas que laboraban como personal asistencial en el Departamento de Estomatología del Hospital Militar Central cuyas edades se encontraban entre 20 a 40 años y de ambos sexos. Se realizó la anamnesis, examen clínico y radiográfico para evaluar la presencia de TTM y de alteraciones de la postura cervical a través del índice de Krogh Poulsen y del Análisis Radiográfico con técnica de Penning respectivamente.
Los resultados indican una prevalencia del 50,9\% de TTM. Las conclusiones son, que la prevalencia con posturales cervical anormal fue de 75,5\% (11).

La discusión de los objetivos específicos, se realizó en función a los indicadores de los trastornos temporomandibulares, de donde tenemos, de acuerdo al objetivo específico número uno que fue: Identificar los signos y síntomas a la apertura máxima bucal se ha encontrado que la apertura "igual o mayor a $40 \mathrm{~mm}$ ", se presenta en un $49,8 \%$ y la apertura bucal "de 30 a 39 mm" 43,8\%, en los estudiantes de la Universidad Privada de Huancayo Franklin Roosevelt, evaluados en el semestre académico 2018-I. Estos resultados en cuanto a porcentaje tienen cierta similitud con lo hallado por Ayala y Carmona, quienes realizaron un estudio en 200 pacientes de ambos sexos de la Clínica Estomatológica "Manuel Ángulo Farrán"- Cuba, los que en cuanto a apertura bucal encontraron restricción en apertura bucal $6,5 \%(12)$.

Según Padilla, aunque su población no fue de músicos encontró dificultad en apertura máxima (igual o mayor de $40 \mathrm{~mm}$ ) en un $78,7 \%$ (10).

En relación al segundo objetivo específico que fue identificar los signos y síntomas en lateralidad derecha máxima bucal se ha encontrado que la lateralidad derecha: "entre 4 a 6 mm" alcanzó el 48\%, la lateralidad derecha "igual o mayor de $7 \mathrm{~mm}$ " obtuvo el $41,9 \%$ y la lateralidad derecha máxima "entre 0 a 3 mm" se presentó en un $10 \%$, en los estudiantes de la Universidad Privada de Huancayo Franklin Roosevelt, evaluados en el semestre académico 2018-I. Según Padilla, aunque su población no fue de músicos encontró lateralidad derecha máxima (igual o mayor de $8 \mathrm{~mm}$ ) en $70,5 \%$ (10).

Continuando con el objetivo específico número tres que dice: Identificar los signos y síntomas en lateralidad izquierda máxima bucal se encontró que la lateralidad izquierda: "igual o mayor de $7 \mathrm{~mm}$ " está presente en un $53,2 \%$, la lateralidad izquierda "entre 4 a $6 \mathrm{~mm}$ " en un $41,3 \%$ y la lateralidad izquierda entre " 0 a $3 \mathrm{~mm}$ " está presente solo en un 5,5\%, en los estudiantes de la Universidad Privada de Huancayo Franklin Roosevelt, evaluados en el semestre académico 2018-I, también según Padilla y en su población de estudiantes de odontología encontró lateralidad izquierda máxima (igual o mayor de $8 \mathrm{~mm}$.) en 63,3\% (10).

Ahora según el cuarto objetivo específico que fue: Identificar los signos y síntomas en protrusión máxima bucal se ha encontrado que la protrusión máxima "entre 
4 a 6 mm" está presente en el $43,8 \%$, la protrusión máxima "igual o mayor de $7 \mathrm{~mm}$ " en el $33,1 \%$ y solo el 23,1\% presentó protrusión máxima "entre 0 a 3 mm" según Índice de Helkimo en los estudiantes de la Universidad Privada de Huancayo Franklin Roosevelt, evaluados en el semestre académico 2018-I. También Padilla, en estudiantes de odontología encontró protrusión máxima (entre 4 - $6 \mathrm{~mm}$ ) en 45,9\% (10).

De acuerdo al objetivo específico cinco que fue: Identificar los signos y síntomas durante el movimiento mandibular se ha encontrado que el movimiento mandibular con "moderado deterioro" está presente en un $58,1 \%$ mientras que el movimiento mandibular con "grave deterioro" está presente en un 32,8\%, según el Índice de Helkimo en los estudiantes de la Universidad Privada de Huancayo Franklin Roosevelt, evaluados en el semestre académico 2018-I.

Respecto al objetivo seis que fue: Identificar los signos y síntomas durante la función de ATM se ha encontrado que los "ruidos articulares o desviación mayor de $2 \mathrm{~mm}$ " está presente en un $51,7 \%$, mientras que "sin ruidos articulares o desviación hasta $2 \mathrm{~mm}$ " alcanzó el 46,2\%, según el Índice de Helkimo en los estudiantes de la Universidad Privada de Huancayo Franklin Roosevelt, evaluados en el semestre académico 2018-I. Los resultados encontrados en la presente investigación difieren de lo hallado por Ayala y Carmona, quienes en cuanto a los signos y síntomas con mayor prevalencia de trastornos témporomandibulares encontraron el ruido articular en un 10\%, en su estudio en 200 pacientes de ambos sexos de la Clínica Estomatológica "Manuel Ángulo Farrán"- Cuba, sometidos a examen clínico para evaluar el tipo de oclusión, los hábitos bucales y trastornos témporomandibulares utilizando la Clasificación de Angle y Test de Helkimo (12).

Resultados similares también tenemos los hallados por Gutiérrez y Gonzáles, quienes evaluaron a 44 pacientes mayores de 18 años de ambos sexos del Servicio de Prótesis de la Clínica Estomatológica Artemio Mastrapa Rodriguez - Cuba, donde aplicaron el Test de Maglione para determinar los trastornos témporomandibulares (TTM) y los grados de severidad que, aunque utilizaron otro índice para TTM, concluyeron que todos los pacientes con trastornos témporomandibulares presentaban las siguientes manifestaciones clínicas: ruido articular en el $81,4 \%$, dolor muscular en $66,7 \%$, limitación en los movimientos en el $37 \%$ (13).
Gonçalves también efectuó un estudio a 40 pacientes de 20 y 40 años del Departamento de Odontología de la Universidad Estatal Paraíba- Brasil. El examen fue extraoral e intraoral con el fin de encontrar trastornos témporomandibulares en los pacientes, concluyeron que un $45 \%$ de pacientes presentaba dolor en las articulaciones y un $12,5 \%$ dolor muscular (7). También tenemos a Delgado, quien evaluó a 41 pacientes del Hospital de Concentración ISSEMYM - México, entre los 19 y 60 años de ambos sexos. Examinó a 32 mujeres (78\%) y 9 hombres (22\%), el examen clínico constó de una evaluación minuciosa, empleando el Test de Helkimo, para identificar la presencia de trastornos témporomandibulares (TTM) más prevalentes, obteniendo como resultado a 33 pacientes con otalgia y crepitación ósea. Concluyendo así que los trastornos témporomandibulares (TTM) son las causas más frecuentes que afectan gravemente la armonía de la ATM (14).

Jiménez examinó a 1201 personas entre 15 años a más de ambos sexos de Ciudad de La Habana. El estudio consistió en anamnesis y examen clínico para diagnosticar trastornos temporomandibulares. Se usó el Índice de Helkimo, llegando a la conclusión que la sintomatología principal fueron los ruidos articulares durante los movimientos de apertura y cierre mandibular (9). Pereira evaluó a 36 pacientes (29 mujeres y 7 hombres) entre 22 y 70 años de ambos sexos del Hospital Ricardo Gutiérrez de La Plata - Argentina. Examinaron la existencia de trastornos temporomandibulares (TTM), mediante el uso del Índice de Helkimo. Obtuvieron que: 61,0\% presentó dolor a la palpación de la articulación temporomandibular (ATM); el 55,5\% presentó chasquidos articulares siendo los signos y síntomas más prevalentes del estudio (15). Padilla, en estudiantes de odontología, encontró función de ATM (ruidos articulares o desviación de mayor de $2 \mathrm{~mm}$ en apertura o cierre) en $80,3 \%$ (10).

En función al objetivo específico que fue: Identificar los signos y síntomas del estado muscular se halló que el estado muscular "sin sensibilidad a la palpación" está presente en un $79 \%$, el estado muscular "sensibilidad a la palpación hasta en tres áreas" está en un 20,1\% y la "sensibilidad en más de tres áreas" obtuvo un $9 \%$, según el Índice de Helkimo en los estudiantes de la Universidad Privada de Huancayo Franklin Roosevelt, evaluados en el semestre académico 2018-I. Del mismo estudio, Padilla encontró en estudiantes de odontología que en estado muscular, sin sensibilidad a la palpación se tuvo $60,6 \%$ (10). 
Continuando con el objetivo número siete: Identificar los signos y síntomas del estado de la articulación temporomandibular se halló que el estado de la articulación temporomandibular "sin sensibilidad a la palpación" está presente en un $75,1 \%$, el estado de la articulación temporomandibular con "sensibilidad a la palpación lateral" en un $24 \%$ y $9 \%$ la "sensibilidad a la palpación posterior", según el indice de Helkimo en los estudiantes de la Universidad Privada de Huancayo Franklin Roosevelt, evaluados en el semestre académico 2018-I. Podemos volver a mencionar a Padilla, quien en estudiantes de odontología encontró en estado de la articulación témporomandibular (ATM), con sensibilidad a la palpación en $57,4 \%$, resultado que difiere de lo hallado en la presente investigación (9).

Respecto al objetivo específico: Identificar los signos y síntomas de dolor durante el movimiento mandibular se mostró que el "movimiento mandibular sin dolor" está en un $70,2 \%$ de la muestra, el "dolor referido a un solo movimiento" obtuvo el $24,1 \%$ y sólo el 5,8\% presentaron "dolor en dos o más movimientos", según el Índice de Helkimo en los estudiantes de la Universidad Privada de Huancayo Franklin Roosevelt, evaluados en el semestre académico 2018I, según lo encontrado la condición de movimiento mandibular sin dolor alcanzó el porcentaje del 97,9\% y la condición de dolor referido a un solo movimiento obtuvo solo el 2,1\%. También Grau y Cabo, quienes evaluaron a 40 pacientes de ambos sexos entre 18 y 45 años del Departamento de Prótesis de la Universidad de Ciencias Médicas de La Habana - Cuba, a través de examen clínico y tomando modelos de estudio para una mejor observación de cada caso, encontraron que los movimientos laterales, protrusión y apertura, dieron como resultado un $92,5 \%$ con dolor muscular y articular, dolor en apertura mandibular a un $87,5 \%$ del total de pacientes. Concluyeron que cada paciente que sufre de trastornos temporomandibulares tiene interferencias oclusales ubicadas específicamente en la zona de no trabajo, encontrando limitaciones en los movimientos de lateralidad y protrusión, impidiendo la ejecución normal de dichos movimientos (16).

De alguna forma también hay similitud a lo encontrado por Ayala y Carmona quienes realizaron un estudio en 200 pacientes de ambos sexos de la Clínica Estomatológica sometidos a examen clínico para evaluar el tipo de oclusión, los hábitos bucales y trastornos temporomandibulares utilizando la Clasificación de Angle y Test de Helkimo, para así determinar maloclusiones, quienes hallaron en cuanto al síntoma dolor muscular el 8\% (12). Podemos volver a mencionar a Padilla, que en su población de estudiantes de odontología encontró dolor al movimiento mandibular (un solo movimiento) 49,2\% (10).

Finalmente, respecto al último objetivo específico: Identificar el trastorno temporomandibular más frecuente, se encontró que de acuerdo a la edad el "trastorno moderado" está presente en un $40,12 \%$ en el grupo de 15 a 34 años, según el sexo en el femenino en un $42,5 \%$ y según la escuela profesional está presente en un 27,4\% en Ciencias Farmacéuticas y Bioquímica.

\section{CONCLUSIONES}

1. Respecto a los trastornos temporomandibulares, se ha encontrado que el "trastorno moderado" es el más prevalente con el $55,9 \%$ de la muestra, seguido del "trastorno severo grado I" con un $38,9 \%$ con 128 datos, en los estudiantes de la Universidad Privada de Huancayo Franklin Roosevelt, evaluados en el semestre académico 2018-I, con el Test de Helkimo.

2. Se ha identificado respecto a los signos y síntomas de la apertura máxima bucal, que la apertura "igual o mayor a $40 \mathrm{~mm}$ " se presenta en un $49,8 \%$ y la apertura bucal "de 30 a 39 mm" $43,8 \%$, en los estudiantes de la Universidad Privada de Huancayo Franklin Roosevelt, evaluados en el semestre académico 2018-I.

3. Se ha identificado respecto a los signos y síntomas de la lateralidad derecha que la lateralidad derecha: "entre 4 a 6 mm" alcanzó el 48\%, la lateralidad derecha "igual o mayor de $7 \mathrm{~mm}$ " obtuvo el $41,9 \%$ y la lateralidad derecha máxima "entre 0 a $3 \mathrm{~mm}$ " se presentó en un $10 \%$, en los estudiantes de la Universidad Privada de Huancayo Franklin Roosevelt, evaluados en el semestre académico 2018-I.

4. Se ha identificado respecto a los signos y síntomas de la lateralidad izquierda que la lateralidad izquierda: "igual o mayor de $7 \mathrm{~mm}$ " está presente en un $53,2 \%$, la lateralidad izquierda "entre 4 a 6 mm" en un $41,3 \%$ y la lateralidad izquierda entre "0 a 3 mm" está presente solo en un 5,5\%, en los estudiantes de la Universidad Privada de Huancayo Franklin Roosevelt, evaluados en el semestre académico 2018-I.

5. Se ha identificado respecto a los signos y síntomas en protrusión máxima bucal que la protrusión máxima "entre 4 a $6 \mathrm{~mm}$ " está presente en el $43,8 \%$, la protrusión máxima "igual o mayor 
de $7 \mathrm{~mm}$ " en el $33,1 \%$ y solo el $23,1 \%$ presentó protrusión máxima "entre 0 a 3 mm" según Índice de Helkimo en los estudiantes de la Universidad Privada de Huancayo Franklin Roosevelt, evaluados en el semestre académico 2018-I.

6. Se ha identificado respecto a los signos y síntomas durante el movimiento mandibular que el movimiento mandibular con "moderado deterioro" está presente en un $58,1 \%$ mientras que el movimiento mandibular con "grave deterioro" está presente en un 32,8\%, según el Índice de Helkimo en los estudiantes de la Universidad Privada de Huancayo Franklin Roosevelt, evaluados en el semestre académico 2018-I.

7. Se ha identificado respecto a los signos y síntomas durante la función de ATM que los "ruidos articulares o desviación mayor de $2 \mathrm{~mm}$ " está presente en un $51,7 \%$, mientras que "sin ruidos articulares o desviación hasta $2 \mathrm{~mm}$ " alcanzó el $46,2 \%$, según el índice de Helkimo en los estudiantes de la universidad privada de Huancayo Franklin Roosevelt, evaluados en el semestre académico 2018-I.

8. Se ha identificado respecto a los signos y síntomas del estado muscular que el estado muscular "sin sensibilidad a la palpación" está presente en un $79 \%$, el estado muscular "sensibilidad a la palpación hasta en tres áreas" está en un 20,1\% y la "sensibilidad en más de tres áreas" obtuvo un 9\%, según el Índice de Helkimo en los estudiantes de la Universidad Privada de Huancayo Franklin Roosevelt, evaluados en el semestre académico 2018-I.

9. Se ha identificado respecto a los signos y síntomas del estado de la articulación, que el estado de la articulación temporomandibular "sin sensibilidad a la palpación" está presente en un $75,1 \%$, el estado de la articulación temporomandibular con "sensibilidad a la palpación lateral" en un $24 \%$ y 9\% la "sensibilidad a la palpación posterior", según el Índice de Helkimo en los estudiantes de la Universidad Privada de Huancayo Franklin Roosevelt, evaluados en el semestre académico 2018-I.

10. Se ha identificado respecto a los signos y síntomas de dolor al movimiento que el "movimiento mandibular sin dolor" está en un $70,2 \%$ de la muestra, el "dolor referido a un solo movimiento" obtuvo el $24,1 \%$ y sólo el $5,8 \%$ presentaron "dolor en dos o más movimientos", según el Índice de Helkimo en los estudiantes de la Universidad Privada de Huancayo Franklin Roosevelt, evaluados en el semestre académico 2018-I.
11. Se ha identificado los trastornos temporomandibulares, según el Índice de Helkimo en los estudiantes de la Universidad Privada de Huancayo Franklin Roosevelt, de acuerdo a la edad el "trastorno moderado" está presente en un $40,12 \%$ en el grupo de 15 a 34 años, según el sexo en el femenino en un 42,5\% y según la escuela profesional está presente en un $27,4 \%$ en Ciencias Farmacéuticas y Bioquímica.

\section{Correspondencia:}

Mg. Luis Alberto Cueva Buendía

Correo electrónico: lcueva@uroosevelt.edu.pe

\section{REFERENCIAS BIBLIOGRÁFICAS}

1. Reyes J, Loera L. Trastornos Témporomandibular. Rev Med Oral. 2008; 10 (2): 56 -59.

2. Ayres H, Acácio E. Clasificación de la articulación temporomandibular: aspectos anátomofuncionales. Int J Odontostomat. 2007; 1(1): 25-28.

3. Okeson JP, Brace H. Tratamiento de la oclusión y las afecciones temporomandibulares. España: Ed. Mosby;1999.

4. Dawson P. Oclusión funcional: diseño de la sonrisa a partir de la ATM. Florida, EUA: Editorial Amolca; 2009.

5. Van der Weele LT, Dibbets JM. Helkimo's index: a scale or just a set of symptoms. J Oral Rehabil. 1998;14(3):229-37.

6. Silva P. Postural evaluation of patients with temporomandibular disorders under use of occlusal splints. J Appl Oral Sci. 2009; 17(5): 539-543.

7. Gonçalves F. Prevalência de sinais, sintomas e fatores associados em portadores de trasntornos témporomandibular. Acta Scientiarum Health Science. 2009;31(2):159-163.

8. Hormiga C. Prevalencia de síntomas y signos de trastornos témporomandibulares en una población universitaria del área metropolitana de Bucaramanga, Santander. Rev Umbral Científico. 2009; 14: 80 - 91.

9. Jiménez Z. Prevalencia de los trastornos témporomandibulares en la población de 15 años y más de la Ciudad de La Habana. Rev Cuba estomatol; 2007; 44(3):0-0.

10. Padilla M. Prevalencia de signos y síntomas de trastornos témporomandibulares y grados según Índice de Helkimo en pacientes adultos atendidos en la Clínica Odontológica de la Universidad Privada Norbert Wiener, Lima 2010.Tesis para optar el título de cirujano dentista. Lima: Universidad Privada Norbert Wiener; 2011.

11. Limaylla R.Trastornos temporomandibulares y alteraciones posturales de la columna cervical en personal asistencial del Departamento de Odontología 
del Hospital Militar Central.Tesis para optar el título de cirujano dentista. Lima: Universidad Nacional Mayor de San Marcos;2007.

12. Ayala Y, Carmona E. Maloclusiones y características clínicas de trastornos temporomandibulares en pacientes de la Clínica Manuel Angulo. Correo Científico Médico de Holguín 2010; 14(2):0-0.

13. Gutiérrez M, González E. Trastornos tem poromandibulares en pacientes bruxópatas atendidos en la Clínica Artemio Mastrapa Rodríguez. Correo Científico Médico de Holguín 2010; 14(3):0-0.
14. Delgado SB, SánchezGA. Manifestaciones Otológicas ante trastornos de la articulación témporomandibular. Anales de Otorrinolaringología Mexicana. 2009; 54 (3): 112-118.

15. Pereira D. Compromiso de la articulación témporomandibular en la artritis reumatoidea. CES Odontología.2001; 14(2): 63-72.

16. Grau I, Cabo R. Evaluación de la oclusión en pacientes con trastornos témporomandibulares y desarmonías oclusales. Rev Cubana de Estomatol. 2010; 47(2):169 -177 .

$\begin{array}{ll}\text { Recibido: } & 12 / 05 / 2019 \\ \text { Aceptado: } & 2 / 12 / 2019\end{array}$

